\title{
Significance of Visible Non-Invasive Risk Attributes for the Initial Prediction of Heart Disease Using Different Machine Learning Techniques
}

\author{
Syed Immamul Ansarullah (D), ${ }^{1}$ Syed Mohsin Saif ${ }^{D},{ }^{2}$ Pradeep Kumar (D), \\ and Mudasir Manzoor Kirmani ${ }^{4}$ \\ ${ }^{1}$ Lecturer at the Department of Computer Science, Cluster University, Jammu, India \\ ${ }^{2}$ Assistant Professor at the Department of Information Technology, IUST Awantipora, Kashmir, India \\ ${ }^{3}$ Associate Professor at the Department of Computer Science and Information Technology, MANUU, Hyderabad, India \\ ${ }^{4}$ Assistant Professor at the Department of Fisheries, SKAUST, Kashmir, India
}

Correspondence should be addressed to Syed Immamul Ansarullah; syedansr@gmail.com

Received 29 October 2021; Revised 11 January 2022; Accepted 19 January 2022; Published 21 February 2022

Academic Editor: Gaurav Singal

Copyright (c) 2022 Syed Immamul Ansarullah et al. This is an open access article distributed under the Creative Commons Attribution License, which permits unrestricted use, distribution, and reproduction in any medium, provided the original work is properly cited.

\begin{abstract}
Introduction. Heart disease is emerging as the single most critical cause of death worldwide and is one of the costliest chronic conditions. Purpose. Stimulated by the increasing heart disease mortality rate incidents, an effective, low-cost, and reliable heart disease risk evaluation model is developed using significant non-invasive risk attributes. The significant non-invasive risk attributes like (age, systolic BP, diastolic BP, BMI, hereditary factor, smoking, alcohol, and physical inactivity) are identified by the help of medical domain experts, and their reliability in heart disease prediction is investigated through different feature selection techniques. Methodology. The enhancements of applying specific investigated techniques like random forest, Naïve Bayes, decision tree, support vector machine, and $\mathrm{K}$ nearest neighbor to the risk factors are tested. The heart disease risk assessment model is developed using the Jupyter Notebook web application, and its performance is tested not only through medical domain measures but also through the model performance measures. Findings. To evaluate heart disease risk evaluation model, we calculated measures of discrimination like error rate, AUROC, sensitivity, specificity, accuracy, precision, and so on. Experimental results show that the random forest heart disease risk evaluation model outperforms other existing risk models with admirable predictive accuracy and minimum misclassification rate. Originality. The heart disease risk evaluation model is developed based on novel non-invasive heart disease dataset, which consists of 5776 records. This dataset is collected from different heterogeneous data sources of Kashmir (India) through quantitative data collection methods. Research Implications. The risk model is applicable where people lack the facilities of integrated primary medical care technologies for untimely heart disease risk prediction. Future Work. To investigate deep learning and study the significance of other controlled attributes on different age and sex groups in the risk estimation of heart disease.
\end{abstract}

\section{Introduction}

Heart disease is the most influential socioeconomic and public health problem, which has potentially affected both genders with a significant number of causalities and other disabilities [1, 2]. Regardless of being among the most widespread chronic condition leading to a large percentage of disability and mortality across the globe, heart disease is recognized to be among the most avoidable and controllable diseases [3]. Initial identification of cardiac disorder victims can benefit from recuperating patients' health and diminishing the death ratio [4]. If we are to reduce the alarming circumstances emerging out from heart disease, it is implicit to recognize its causal factors that have pushed the world to an unfavorable situation [5]. It is widely accepted that risk factors like age, harmful intake of alcohol, unhealthy diet, 
smoking, and stagnation are the significant risk attributes of heart disease and continuing exposure to these risk attributes results in raised hypertension, diabetes, dyslipidemia, obesity, and stroke [6-12]. The initial prediction of heart disease decreases advancement to critical conditions and complexities [13, 14]. Hence, keeping in view its consequences, we developed a heart disease risk assessment model using machine learning techniques, which would help physicians in initial prediction with high predictive power.

\section{Literature Review}

In recent times, researchers made decisive contributions to predict heart disease using different machine learning techniques. Palaniappan and Awang [15] applied Naive Bayes, neural network, and decision tree on patient attributes and evaluated model's performance using lift chart and classification matrix. However, the model is only used by stake holders. Anooj [16] applied a weighted fuzzy rule to develop risk model and then evaluated its performance through neural network based system on UCI heart disease dataset. Taneja [17] collected transthoracic echocardiography dataset and applied J48 classifier, Naive Bayes, and multilayer perceptron to get the significant risk attributes. These researchers analyzed the model results, and it is observed that the model has optimal accuracy and high specificity rate. Sujata and Nair [18] applied decision tree, Naïve Bayes, and K nearest neighbor techniques for initial prediction of heart disease. Purushottam et al. [19] applied support vector machine, C4.5, neural network, PART, multilayer perceptron, and radial basis function to find out the relationship between several patients and to find out the cause of heart disease. Kim and Kang [20] collected Korean heart disease dataset of 4146 records and applied neural network using feature correlation analysis to identify significant risk attributes and identify existence of correlations between feature relations. The proposed model outperformed Framingham risk score. Haq et al. [21] developed hybrid heart disease model on Cleveland dataset and applied classification algorithms and selection algorithms to select important attributes. They used 10-fold cross-validation for system validation and checked the performance of the classifiers using seven different evaluation metrics. Shah et al. [22] collected Z-Alizadeh Sani from Iranian patients and applied ten machine learning algorithms to predict heart disease. They introduced N2Genetic optimizer that provided optimal accuracy and F-1 score while predicting heart disease risk. Budholiya et al. [23] proposed an approach to identify important heart disease risk attributes using mean Fisher based feature selection algorithm and accuracy based feature selection algorithm. Researchers used principal component analysis to refine the selected feature subset, and the resulting feature subset is used for the classification purpose through RBF-based support vector machine (SVM). Martins et al. [24] applied Bayesian optimization XG boost classifier and one-hot encoding technique to predict heart disease. The performance of the model is evaluated on Cleveland heart disease dataset, and the results are compared with different existing models. Barik et al. [25] applied decision tree, optimized decision tree, random forest, and other algorithms to predict heart disease at its initial stages. These risk models were developed using RapidMiner and WEKA tool and were analyzed based on accuracy, precision, sensitivity, and specificity. Though different methods and algorithms were used to predict heart disease with optimal accuracy in state-of-the-art research; however, some performed with less efficiency. Our research focus on identifying the significant non-invasive heart disease risk attributes by using different feature selection techniques and classification algorithms. The results obtained are a measure to indicate how these techniques can efficiently be used in medical field.

\section{Research Design for Heart Disease Risk Evaluation Model}

To build an efficient heart disease risk evaluation model, we formulate the research design which is described in Figure 1.

The proposed research design consists of four main phases.

3.1. Data Phase. The data phase contains the whole process from data collection to feature engineering. This phase includes the qualitative data collection, the preprocessing subsystem, the cleaned data set storage, and the feature selection step. In this phase, the basic statistical description is performed to learn about each attribute value of the heart disease dataset. The heart disease dataset consists of a combination of nominal and numeric risk attributes. The missing numeric values are removed through the simple mean imputation method, and categorical missing values are filled by mode imputation technique. We performed class balance test on the heart disease dataset because highly imbalanced data makes the machine learning algorithms biased. After analysis, it is found that the heart disease dataset is balanced and has a skewness of $(-0.03065287)$ and kurtosis of $(-2.000136)$. These values of skewness and kurtosis represent that the heart disease dataset values are normally distributed.

The dataset contains 5776 records, of which 2745 (47.5\%) have heart disease, and 3031 (52.5\%) are healthy. Heart disease affects both men and women approximately in the same proportion with substantial death rates and disabilities, and predicting it accurately constitutes several basic causes like social, commercial, and cultural transition. The long-term disclosure to these risk attributes affects the hardest and ends up in death.

3.1.1. Finding Correlation among Different Heart Disease Risk Attributes. In this research, Pearson's correlation is applied to check the mutual relationship among the heart disease attributes. The result of the applied Pearson's correlation coefficients among the heart disease risk attributes is shown in Figure 2 in the form of heatmap representation. The heatmap grid represents the correlation between the heart disease attributes with their corresponding coefficients. After heatmap analysis, we found that independent attributes are loosely 


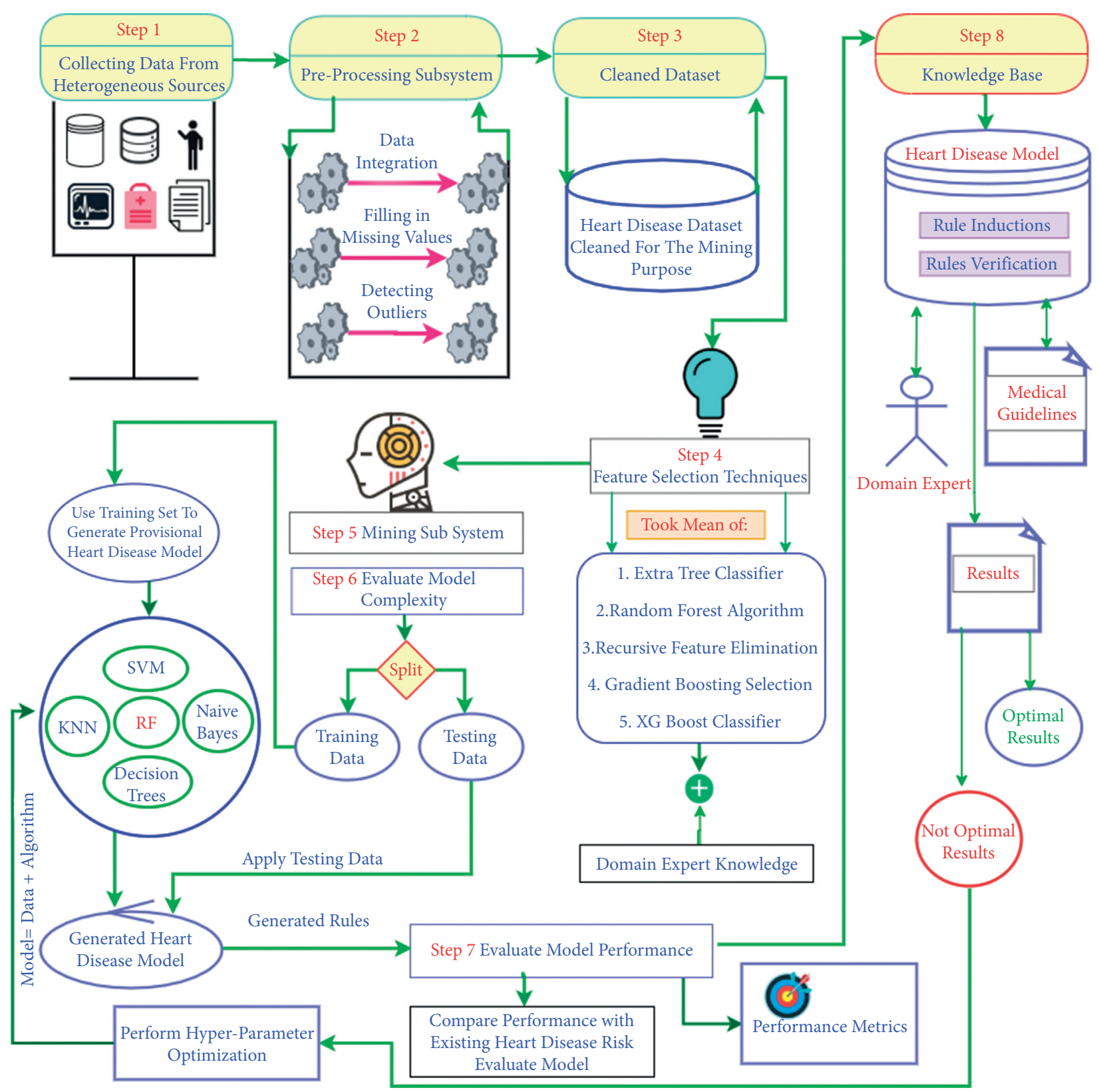

FIgURE 1: Research design for initial heart disease risk prediction.

correlated with one another, which are a good sign to improve the performance of the model. However, if the attributes in a dataset are tightly correlated (called multicollinearity), then a change in one variable can lead to change to another variable that can deteriorate the performance of an algorithm. Correlation among the attributes does not mean causation; hence, the strong relationship among attributes should be evaluated significantly. Mostly, a relationship among attributes may look causal through strong correlation because of some overlooked factors.

3.1.2. Feature Selection Techniques for Heart Disease Risk Assessment. In this study, filter, wrapper, and embedded feature selection methods are applied to get an appropriate subset of feature for initial heart disease risk evaluation. The five different feature elimination techniques (extra tree classifier, gradient boosting classifier, random forest, recursive feature elimination, and XG boost classifier) are used as shown in Figure 3.

Each risk attribute is weighted by these feature selection techniques as per their role in disease prediction. The applied feature selection techniques provide weight in between the scale of 0 to 1 to each risk attribute. The risk attribute with the mean value near to 1 are important and attributes with values near to 0 are less significant in predicting heart disease.

Table 1 show the different non-invasive heart disease risk attributes with their respective weights assigned by different feature selection techniques and the overall mean of all the techniques. These heart disease risk attributes were identified 


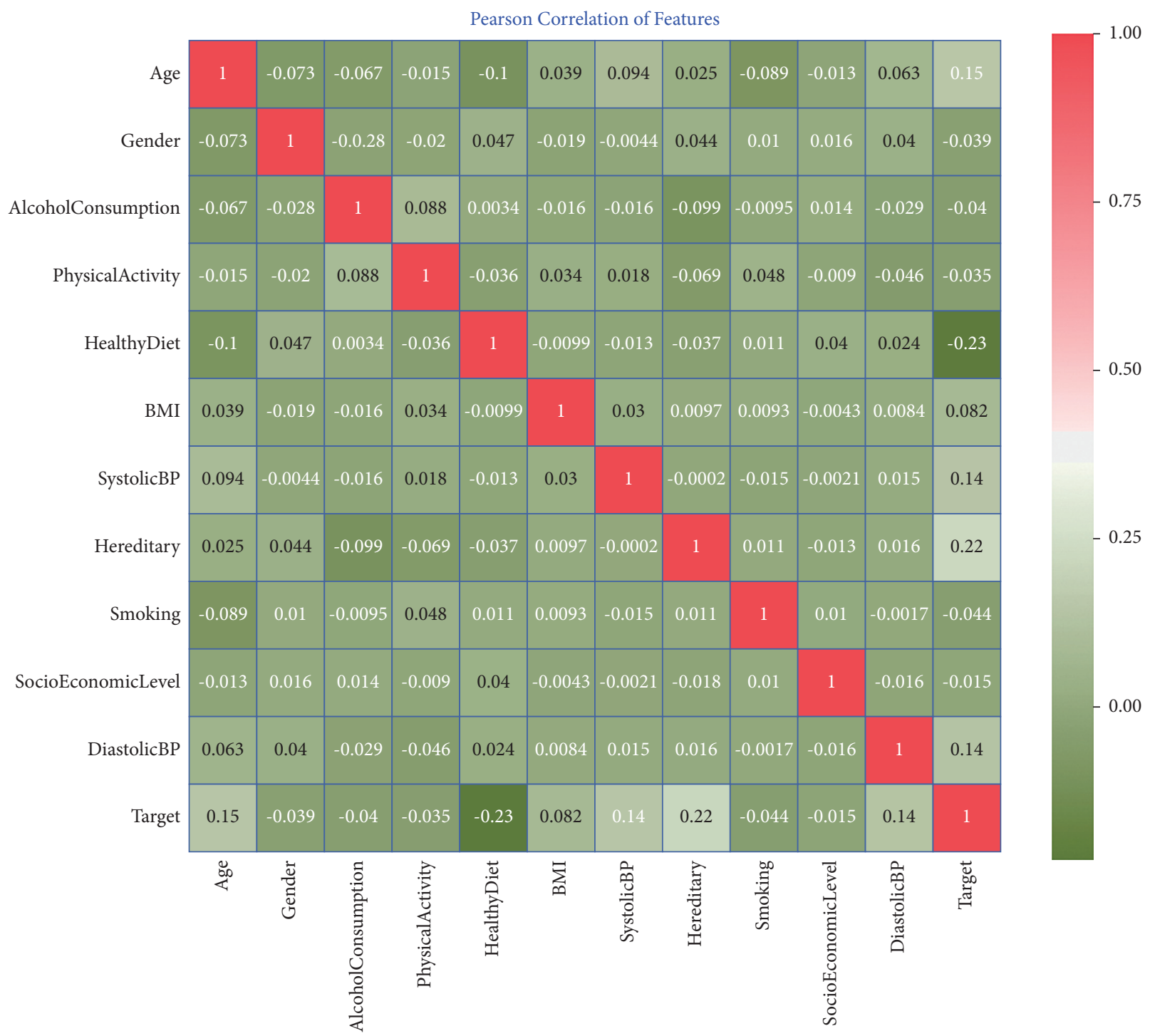

Figure 2: Correlation among different heart disease risk attributes.

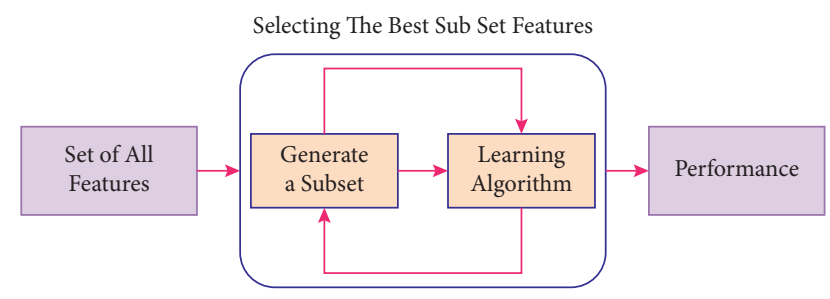

FIGURE 3: Working of feature selection techniques to select the best subset of features.

by professional cardiologists and many other general physicians who are working in the cardiology department at various hospitals across, India.

After analyzing the results, it is derived that the attributes (systolic BP, diastolic BP, age, BMI, hereditary, healthy diet, and physical activity) are the most significant for the early prediction of the heart disease. The highly weighted significant subset of risk features is used to develop the heart disease risk model.
3.2. Data Mining Phase. The heart disease dataset is mined through random forest, decision tree, support vector machine, $\mathrm{K}$ nearest neighbor, and Naive Bayes techniques with 10 -fold cross-validation. Various medical and model domain performance metrics like sensitivity, specificity, accuracy, precision, AUROC score, misclassification rates, computational complexity, and comprehensibility are calculated to obtain the optimal and accurate results. The following subsections explain the experimental results obtained by different heart disease risk evaluation models.

\subsection{Model Evaluation and Validation Phase}

3.3.1. Experimental Results of Decision Tree Model. The rationale to apply a decision tree is to develop a heart disease risk evaluation model that can predict a class (diseased or healthy) by learning simple decision rules deduced from training data [25]. The cross-validation on the training dataset is used to get the unbiased results [26]. The 
TABLE 1: Feature selection techniques providing weight to each risk attribute.

\begin{tabular}{|c|c|c|c|c|c|c|}
\hline \multirow{2}{*}{ Attributes } & \multicolumn{6}{|c|}{ Feature selection techniques with their results and mean values } \\
\hline & ETC & GBC & RF & RFE & XGB & MEAN \\
\hline Age & 0.92 & 0.92 & 0.87 & 0.25 & 0.92 & 0.78 \\
\hline Sex & 0.0 & 0.0 & 0.11 & 0.83 & 0.0 & 0.19 \\
\hline Alcohol consumption & 0.09 & 0.09 & 0.09 & 0.75 & 0.09 & 0.22 \\
\hline Physical activity & 0.25 & 0.25 & 0.08 & 0.67 & 0.25 & 0.30 \\
\hline Healthy diet & 0.71 & 0.71 & 0.52 & 1.0 & 0.71 & 0.73 \\
\hline $\mathrm{BMI}$ & 0.74 & 0.74 & 0.79 & 0.0 & 0.74 & 0.60 \\
\hline Hereditary & 0.38 & 0.38 & 0.4 & 0.92 & 0.38 & 0.49 \\
\hline Smoking & 0.17 & 0.17 & 0.09 & 0.5 & 0.17 & 0.22 \\
\hline Systolic BP & 1.0 & 1.0 & 1.0 & 0.08 & 1.0 & 0.82 \\
\hline Diastolic BP & 0.88 & 0.88 & 0.78 & 0.33 & 0.88 & 0.75 \\
\hline Socio-economic level & 0.17 & 0.17 & 0.11 & 0.42 & 0.17 & 0.21 \\
\hline
\end{tabular}

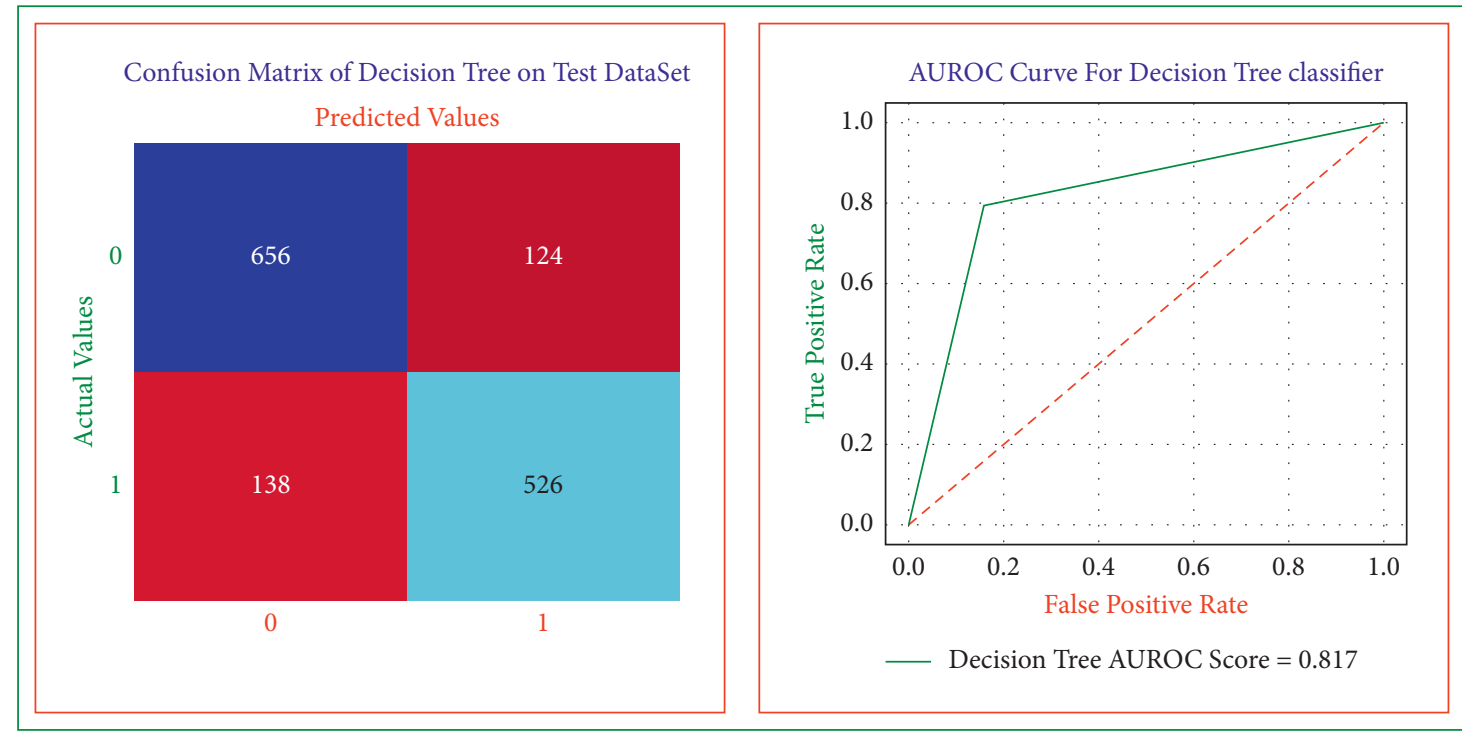

FIgURE 4: Decision tree confusion matrix and AUROC curve on the test dataset.

performance results like sensitivity, specificity, accuracy, precision, error rates, and AUROC score are derived using decision tree model (Figure 4.

The sensitivity [26] of decision tree heart disease model is equal to 0.82 . The closer the value for this measure is to 1 , the better the rules are at identifying those patients who have heart disease. The specificity [27] of the model is equal to 0.80 . The nearer the value for this measure is to 1 , the best the rules are at identifying those patients without the disease. The overall accuracy of the decision tree model is equal to 0.81 , which represents the decision tree heart disease model's overall performance (in diagnosing both the diseased and nondiseased heart disease cases). If accuracy of the model is high, then the model is more accurate in predicting the healthy and diseased cases. The precision is equal to 0.84 . The closer the value for this measurement is to 1 , the greater the chance that those with a positive outcome will have a disease. If a high precision rate of the decision tree model is obtained, then it means that the model will obtain a low false-positive rate. The error rate of this decision tree model is equivalent to 0.18 . The lower the percentage of misclassification rate of the model is, the more accurate the model is in identifying the diseased and healthy cases. The AUROC score is equal to 0.81 . The area under a correlation curve plotting true positive against false positive is higher for models best able to correctly identify positive and negative cases.

3.3.2. Experimental Results of K-Nearest Neighbor Model. The purpose of using the $\mathrm{K}$ nearest neighbor algorithm is to develop a risk evaluation model that can predict heart disease at its earliest. We used the 10-fold cross-validation on training data to get optimal and unbiased results $[28,29]$. Figure 5 shows the K-NN heart disease model, and by using this, we obtained the sensitivity, specificity, accuracy, precision, error rate, and AUROC score equal to 0.73, 0.66, 0.69, $0.69,0.30$, and 0.70 , respectively.

3.3.3. Experimental Results of Support Vector Machine Model. In this research, we used support vector machine model to predict heart disease in its early stages $[30,31]$. The heart disease SVM model is shown in Figure 6 and using this 


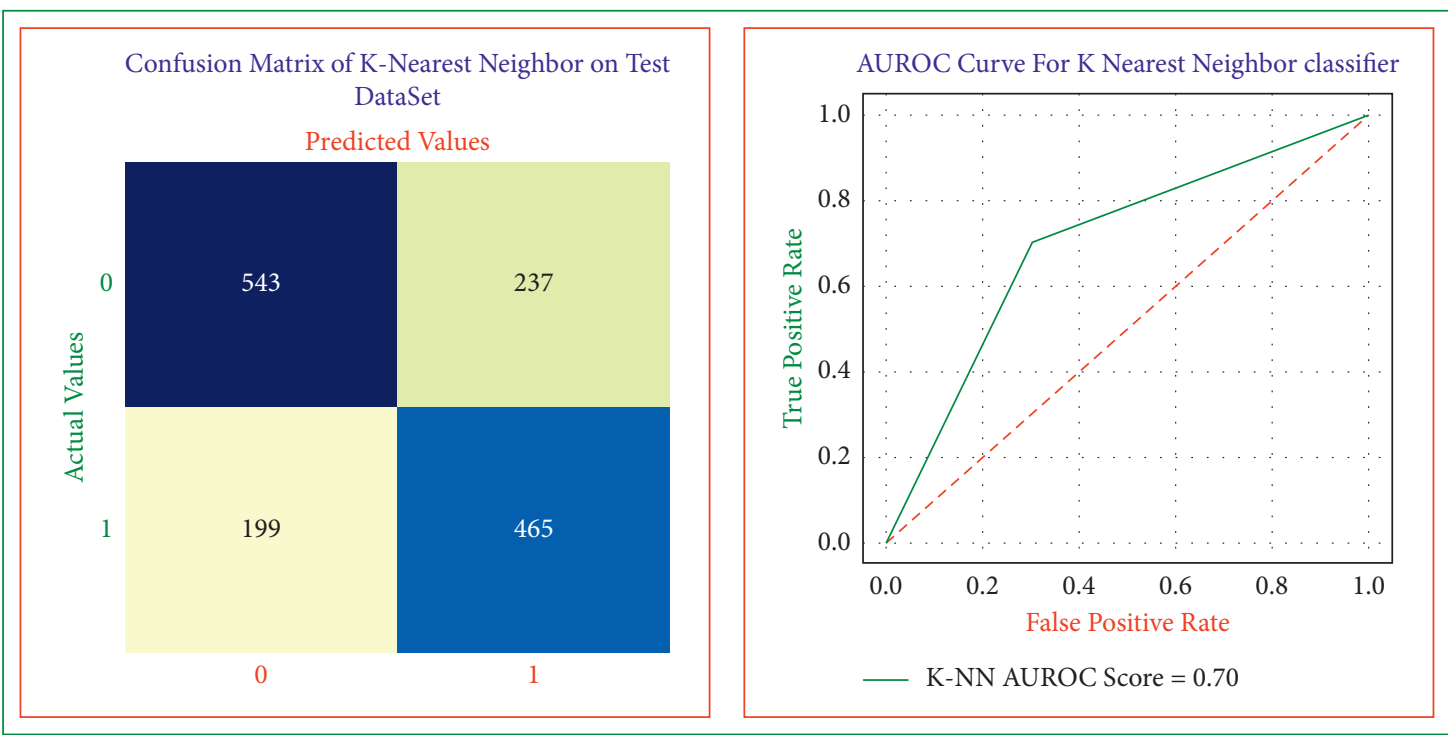

FIgURE 5: K nearest neighbor confusion matrix and AUROC curve on the test dataset.

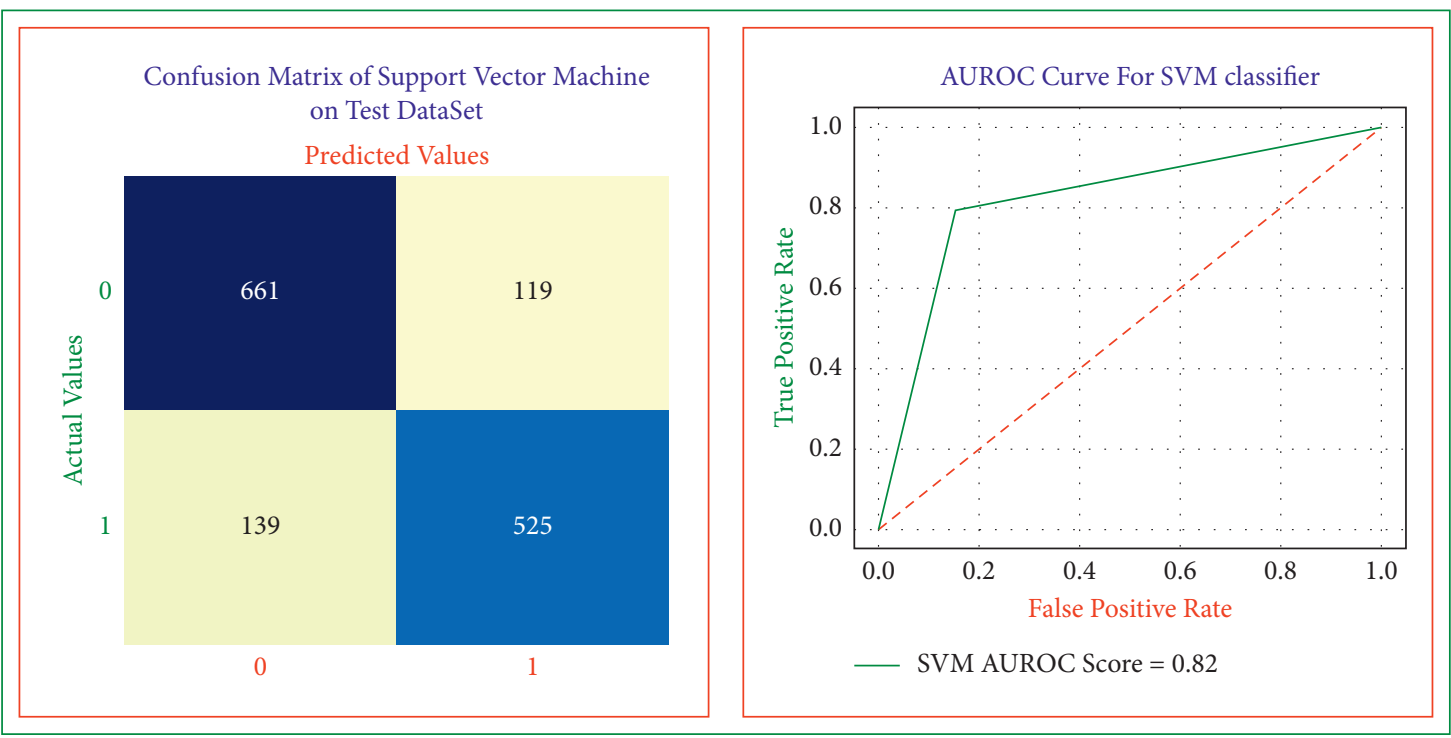

FIgURE 6: SVM risk model confusion matrix and AUROC on test dataset.

model, we obtained the sensitivity, specificity, accuracy, precision, error rates, and AUROC score equal to 0.82, 0.81, $0.82,0.84,0.17$, and 0.82 , respectively.

3.3.4. Experimental Results of Random Forest Model. The predictive results of the heart disease random forest model are shown in Figure 7 [32]. The random forest model recognizes different patient cases with a sensitivity of 0.85 , specificity of 0.83 , accuracy of 0.84 , precision of 0.85 , error rate of 0.15 , and AUROC score of 0.85 .

3.3.5. Experimental Results of Naive Bayes Model. Naive Bayes risk evaluation model is shown in Figure 8 which is used to predict the heart disease at its initial stages. We applied 10-fold cross-validation on heart disease dataset to achieve the maximum accuracy and unbiased results. The performance results of the Gaussian Naive Bayes risk model are sensitivity equal to 0.72 , specificity equal to 0.66 , the overall accuracy equal to 0.69 , precision equal to 0.70 , error rate equal to 0.30 , and AUROC score equal to 0.70 .

3.4. Knowledge-Based Phase. The knowledge-based phase includes the steps to store and retrieve knowledge about heart disease. The generated heart disease risk rules would be stored in the knowledge base and cross-checked as per medical guidelines and domain expertise knowledge. The developed heart disease risk evaluation model is innovative because it identifies the degree of risk of heart disease patients using only the non-invasive risk attributes, thus supporting its application as a public screening test. For simplicity, we have called this model heart disease risk 


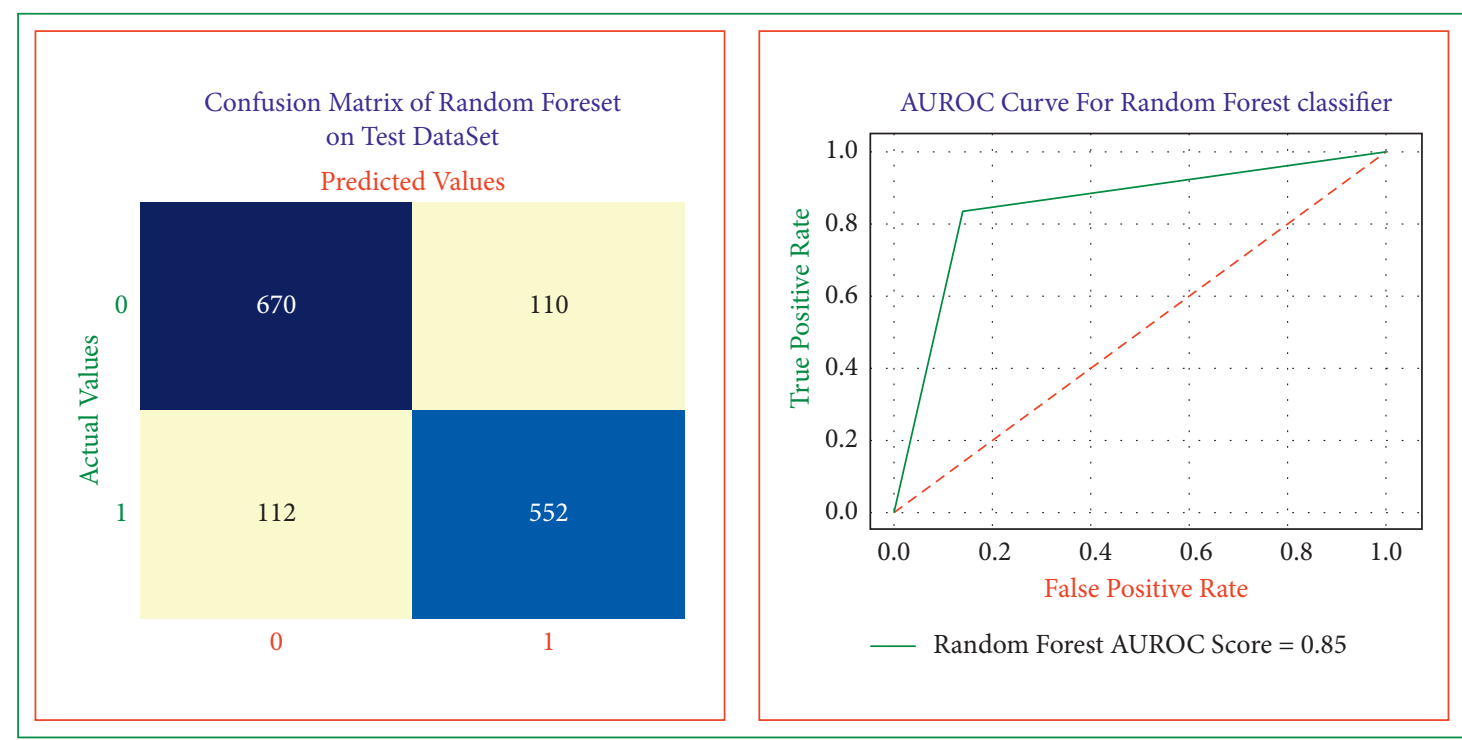

FIGURE 7: Random forest model confusion matrix and AUROC on test dataset.

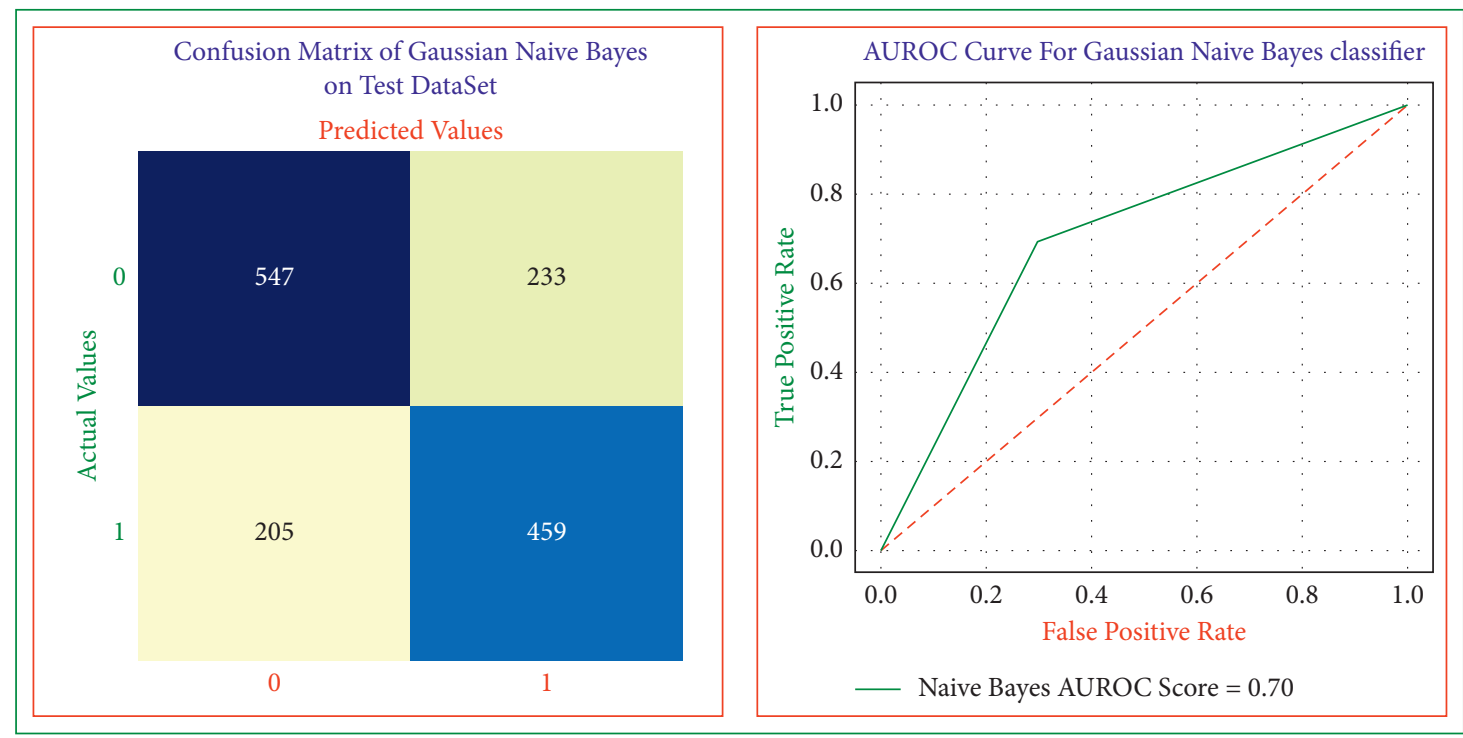

Figure 8: Naive Bayes model confusion matrix and AUROC on test dataset.

evaluation model (HDREM). Figure 9 shows three main components of HDREM and their working: the knowledge base, inference engine, and the interface.

The knowledge base component applies the proposed models on non-invasive heart disease data attributes to extract the expert system rules. The inference engine uses the extracted rules, and the users' input component draws conclusions from the knowledge base and presents them to the user via the user interface. The user interface allows for "communication" screens where the user enters input data, and the expert system returns the degree of heart disease risk as calculated by the inference engine.

The results demonstrate that the combination of age, systolic BP, diastolic BP, BMI, healthy diet, hereditary, and physical activity provides the best results. The rules are extracted to create a chart as community screening tests to support healthcare experts predict the degree of risk of heart disease patients. An optimal set of predictive risk rules are generated using the above-derived attribute combinations, which help in the initial prediction of heart disease victims. The generated heart disease risk evaluation rules are pruned, evaluated, and validated by different medical domain experts; however, their use is restricted as the extracted rules are inductive because they are based on the specific ethnic heart disease dataset.

\section{Consolidated Results of the Risk Model}

We simulate the accomplished experimental results of the developed heart disease risk models with the prevailing research; the results obtained are the best based on the study 


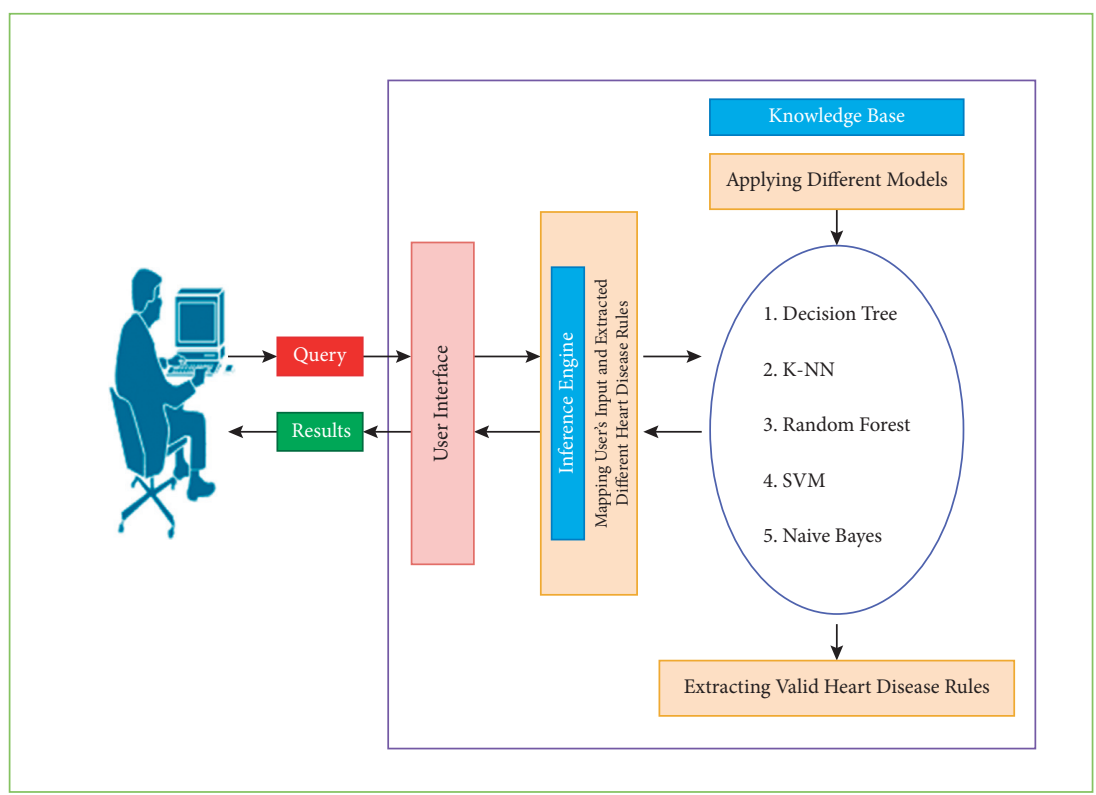

Figure 9: Heart disease expert system evaluation tool components.

TABle 2: Performance measures of developed heart disease models.

\begin{tabular}{lcccccc}
\hline & \multicolumn{5}{c}{ Performance measures } \\
Models & Sensitivity (\%) & Specificity (\%) & Accuracy (\%) & Precision (\%) & Error rate (\%) & AUROC (\%) \\
\hline Decision tree & 82 & 80 & 81 & 84 & 18 & 81 \\
K nearest neighbor & 73 & 66 & 70 & 69 & 30 & 70 \\
Support vector machine & 82 & 81 & 82 & 84 & 82 & 17 \\
Random forest & 85 & 83 & 84 & 85 & 85 \\
Naive Bayes & 72 & 66 & 69 & 70 & 30 & 70 \\
\hline
\end{tabular}

conducted than the published results in the literature. However, there are some exceptions to every proposed heart disease risk assessment model, which are described as follows.

(i) In decision tree risk model, the derived decision tree rules are complex and large, which increases the time complexity of the risk evaluation model and makes the system slow.

(ii) The K nearest neighbor model is not optimal for risk prediction because the misclassification rate is high. The computational complexity and the comprehensibility of the developed heart disease risk model are also high.

(iii) Naive Bayes model is not the best for predicting heart disease because the misclassification rates are higher than the existing proposed models in the literature. Apart from medical domain performance measures, the computational complexity and the comprehensibility of the developed Naive Bayes model are high. The higher values of misclassification rate and model complexity factors restrain its applications because medical prediction models must satisfy greater prediction accuracy and a single misdiagnose can lead to severe consequences.
We also describe the performance and comparison of the proposed risk prediction models through different measures as described in Table 2. Experimental results demonstrate that the random forest model performs most excellent compared with other risk models. The performance of the developed heart disease risk evaluation model is tested with the prevailing risk tools, which demonstrate that the results are exceptionally encouraging with outstanding predictive accuracy. The results show that the random forest model outperforms other risk evaluation models with an optimal accuracy of $85 \%$, specificity of $83 \%$, sensitivity of $85 \%$, precision of $85 \%$, AUROC score of $85 \%$, and with less misclassification rate of only $13 \%$. The accuracy obtained by the random forest is highest for predicting heart disease and is not achieved by previous studies.

Figure 10 shows the combined AUROC curves of different developed heart disease risk evaluation models. The random forest risk evaluation model has the highest AUROC score of 0.85 , which means the model is highly skillful in predicting the diseased and healthy patients.

\section{Results of the Non-Invasive Heart Disease Risk Attributes}

Table 3 demonstrates the performance of various combinations of non-invasive risk attributes in early heart disease predictions. The combinations of systolic BP, diastolic BP, 


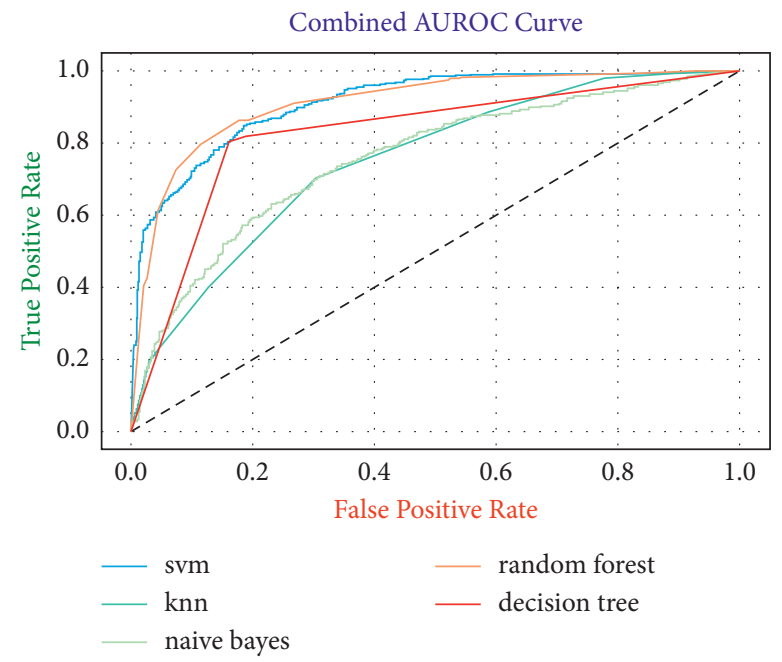

Figure 10: Combined AUROCs of the developed risk evaluation models.

TABLE 3: Integrating different non-invasive heart disease risk factors.

\begin{tabular}{|c|c|c|c|c|}
\hline Techniques & Risk attributes & $\begin{array}{l}\text { Sensitivity } \\
(\%)\end{array}$ & $\begin{array}{l}\text { Specificity } \\
(\%)\end{array}$ & $\begin{array}{l}\text { Accuracy } \\
(\%)\end{array}$ \\
\hline \multirow{8}{*}{ Decision tree } & Systolic BP, diastolic BP, age, heredity & 78 & 80 & 77.3 \\
\hline & Systolic BP, diastolic BP, age, BMI & 72 & 70 & 70.9 \\
\hline & Age, healthy diet, BMI & 68 & 61 & 63.3 \\
\hline & Systolic BP, diastolic BP, age, physical activity & 53 & 60 & 58.6 \\
\hline & Healthy diet, BMI, physical activity, age & 58 & 41 & 50.9 \\
\hline & Healthy diet, physical activity, age, systolic BP, diastolic BP & 45 & 43 & 42.5 \\
\hline & Physical activity, age, healthy diet, BMI, systolic BP, diastolic BP & 38 & 30 & 38.2 \\
\hline & $\begin{array}{c}\text { Age, physical activity, smoking, systolic BP, diastolic BP, healthy diet, alcohol } \\
\text { consumption, BMI }\end{array}$ & 30 & 28 & 42.7 \\
\hline \multirow{6}{*}{ K nearest neighbor } & Age, healthy diet, alcohol consumption, smoking & 42 & 45 & 38.2 \\
\hline & Age, BMI, healthy diet & 70 & 60 & 67.9 \\
\hline & Age, BMI, alcohol consumption, smoking, sex & 52 & 50 & 48.9 \\
\hline & BMI, systolic BP, diastolic BP, age, physical activity & 38 & 35 & 42.7 \\
\hline & BMI, systolic BP, diastolic BP, age & 68 & 74 & 72.5 \\
\hline & Age, systolic BP, BMI, diastolic BP, heredity & 68 & 70 & 72.8 \\
\hline \multirow{5}{*}{ Random forest } & Systolic BP, diastolic BP, age, healthy diet, smoking & 51 & 48 & 45.4 \\
\hline & BMI, age, systolic BP, diastolic BP, heredity & 72 & 78 & 78.9 \\
\hline & $\begin{array}{c}\text { Alcohol consumption, physical activity, age, systolic BP, diastolic BP, BMI, } \\
\text { smoking, healthy diet }\end{array}$ & 35 & 45 & 58.7 \\
\hline & Age, sex, physical activity, BMI, & 32 & 34 & 40.8 \\
\hline & Age, sex, physical activity, BMI, systolic BP, diastolic BP & 39 & 45 & 42.6 \\
\hline \multirow{6}{*}{$\begin{array}{l}\text { Support vector } \\
\text { machine }\end{array}$} & Systolic BP, diastolic BP, age & 72 & 62 & 76.1 \\
\hline & Systolic BP, diastolic BP, age, BMI, heredity & 70 & 78 & 75.2 \\
\hline & Healthy diet, age, BMI & 41 & 53 & 50.9 \\
\hline & Systolic BP, diastolic BP, age, BMI, physical activity & 50 & 44 & 51.6 \\
\hline & BMI, physical activity, alcohol consumption, age & 49 & 50 & 52.4 \\
\hline & Age, alcohol consumption, BMI, healthy diet & 41 & 59 & 52.2 \\
\hline \multirow{4}{*}{ Naive Bayes } & Systolic BP, diastolic BP, age & 74 & 78 & 75.1 \\
\hline & Age, alcohol consumption, healthy diet, sex, BMI & 40 & 44 & 48.8 \\
\hline & Systolic BP, diastolic BP, age, BMI, heredity & 68 & 75 & 77.2 \\
\hline & $\begin{array}{l}\text { Systolic BP, diastolic BP, alcohol consumption, heredity, age, BMI, smoking, } \\
\text { healthy diet, sex, physical activity, }\end{array}$ & 46 & 51 & 50.6 \\
\hline
\end{tabular}

heredity, and age show the best accuracy of $77.3 \%$ obtained by the decision tree model. We also measure the sensitivity and specificity of all the attribute combinations. Here, sensitivity is most effective in diagnosing sick cases to provide proper care. By adding BMI (height and weight) attribute with the combination of (age, systolic BP, diastolic 


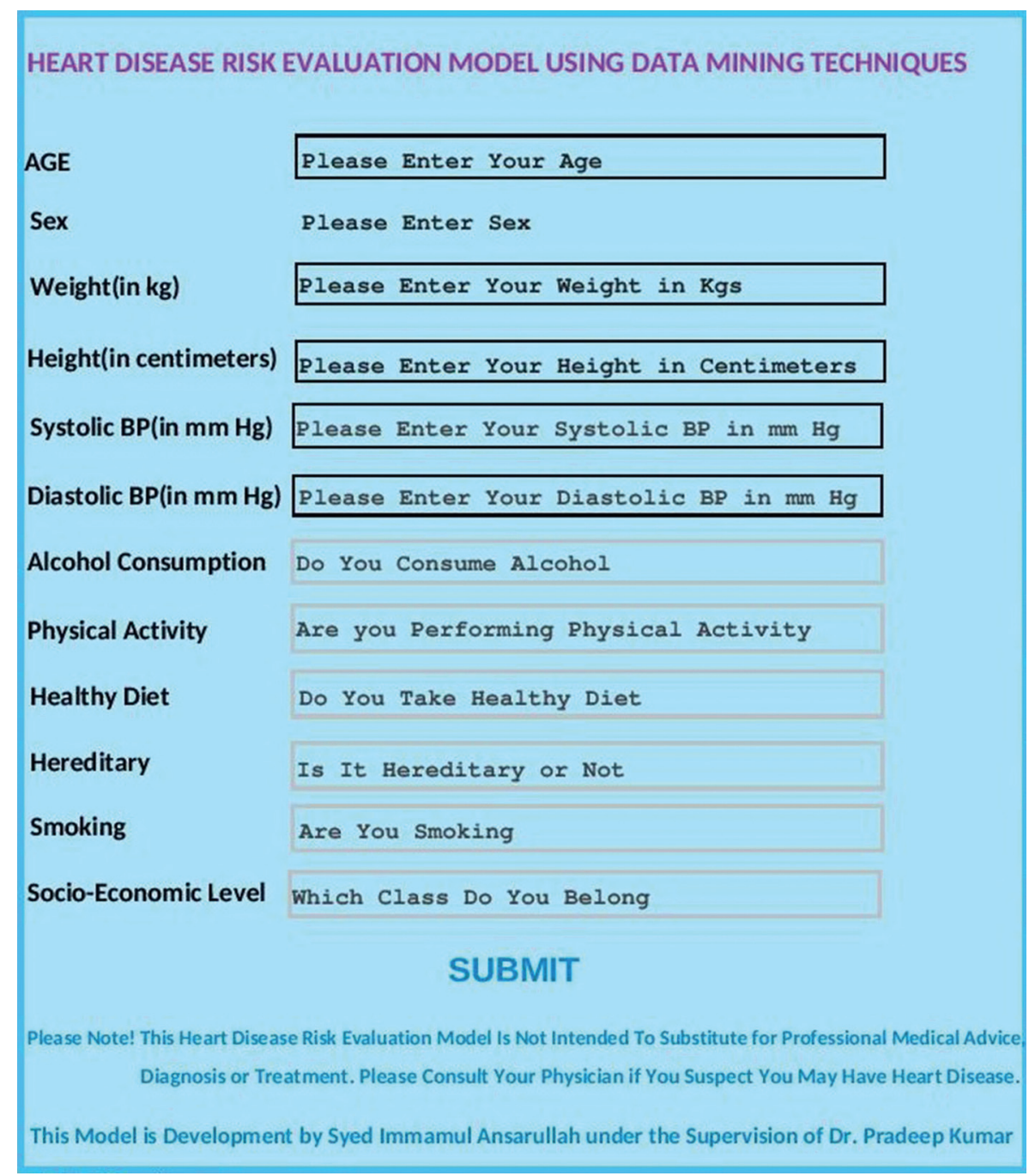

Figure 11: Heart disease risk evaluation model interface.

$\mathrm{BP}$, and heredity), risk features accuracy is increased up to $78.9 \%$ by the random forest model. However, further combinations of the risk attributes with different permutations and combinations decrease the accuracy.

The results demonstrate that the combination of age, systolic BP, diastolic BP, BMI, healthy diet, hereditary, and physical activity provides the best results. The rules are extracted to create a chart as community screening tests to support healthcare experts diagnose the degree of risk of heart disease patients.

The developed model is implemented using the Python Jupyter Notebook web application. Figure 11 shows the start screen of the model, where the user enters his/her data, and based on data, the degree of heart disease risk is calculated and displayed.

The simplicity of the user interface allows health care practitioners to identify patients at high risk of heart disease using very low-cost non-invasive attributes. The model is implemented on mobile as well as desktop applications.

\section{Conclusion}

We developed a non-invasive risk evaluation model that helps in the initial prediction of heart disease. The important and significant risk attributes are selected through careful analysis by cardiologists and different feature selection techniques. After weight assignment to every risk attribute through this process, the overall mean of all attribute weights is considered for the development of heart disease risk model. The higher numeric weight to an attribute is significant and plays a crucial role in predicting heart disease patients at its initial stage. Finally, data mining techniques use weighted risk attributes in predicting and diagnosing heart disease patients. The heart disease dataset is mined using the random forest, $\mathrm{K}$ nearest neighbor, support vector machine, decision tree, and Naive Bayes classifiers to discover if an individual possessing certain modifiable risk features will have the heart disease or not. The specificity, sensitivity, precision, accuracy, misclassification rate, and AUROC scores are calculated for each method using out-of- 
sample testing to check how accurately the risk evaluation model performs. Experimental results show that the random forest model outperforms other models with the highest sensitivity, specificity, precision, accuracy, AUROC score, and minimum misclassification rate. We simulate the accomplished outcomes against the prevailing research; the results obtained are, to the best of our perception, greater than published values in the literature. This risk model is applicable where people lack the facilities of the integrated primary medical care technologies for untimely heart disease risk prediction.

\section{Future Work}

In future, we can enhance the model using the following.

(i) The proposed research could be enhanced by investigating the performance of other robust machine learning techniques like deep learning

(ii) The risk model could be enhanced by adding other non-invasive attributes

(iii) The risk model could give optimal results by identifying the significance of controlled non-invasive attributes, such as weight and smoking on different age and sex groups in the risk estimation of heart disease

\section{Data Availability}

The heart disease risk data used to support the findings of this study are included within the supplementary information file.

\section{Conflicts of Interest}

The authors declare that they have no conflicts of interest.

\section{Supplementary Materials}

The heart disease risk data used to support the findings of this study are included within the supplementary information file. Data of the heart disease risk are in the supplementary section. (Supplementary Materials)

\section{References}

[1] S. S. Virani, Heart Disease and Stroke Statistics-2020 Update: A Report from the American Heart Association, Lippincott Williams and Wilkins, 2020.

[2] A. H. Association, Heart Disease and Stroke Statistics Update Fact Sheet American Heart Association Research Heart Disease, Stroke and Other Cardiovascular Diseases, Coronary Heart Disease (CHD), 2021.

[3] K. M. Sturgeo, "A population-based study of cardiovascular disease mortality risk in US cancer patients," European Heart Journal, vol. 40, no. 48, 2019.

[4] S. C. Larsson, M. Bäck, J. M. B. Rees, A. M. Mason, and S. Burgess, "Body mass index and body composition in relation to 14 cardiovascular conditions in UK Biobank: a Mendelian randomization study," European Heart Journal, vol. 41, no. 2, pp. 221-226, 2020.
[5] R. Alizadehsani, M. Abdar, M. Roshanzamir et al., "Machine learning-based coronary artery disease diagnosis: a comprehensive review," Computers in Biology and Medicine, vol. 111, no. June, p. 103346, 2019.

[6] M. Abdar, W. Książek, U. R. Acharya, R. S. Tan, V. Makarenkov, and P. Pławiak, "A new machine learning technique for an accurate diagnosis of coronary artery disease," Computer Methods and Programs in Biomedicine, vol. 179, p. 104992, 2019.

[7] J. H. Joloudari, "Coronary artery disease diagnosis; ranking the significant features using a random trees model," International Journal of Environmental Research and Public Health, vol. 17, no. 3, pp. 1-24, 2020.

[8] A. Mustaqeem, S. M. Anwar, A. R. Khan, and M. Majid, “A statistical analysis based recommender model for heart disease patients," International Journal of Medical Informatics, vol. 108, no. October, pp. 134-145, 2017.

[9] M. S. Amin, Y. K. Chiam, and K. D. Varathan, "Identification of significant features and data mining techniques in predicting heart disease," Telematics and Informatics, vol. 36, pp. 82-93, 2019.

[10] L. S. Mehta, K. E Watson, A Barac et al., "Cardiovascular disease and breast cancer: where these entities intersect: a scientific statement from the American heart association," Circulation, vol. 137, no. 8, p. e30, 2018.

[11] A. Timmi, "European society of cardiology: cardiovascular disease statistics 2017," European Heart Journal, vol. 39, no. 7, 2018.

[12] D. K. Arnett, "ACC/AHA guideline on the primary prevention of cardiovascular disease: a report of the American college of cardiology/American heart association task force on clinical practice guidelines," Journal of the American College of Cardiology, vol. 74, no. 10, 2019.

[13] M. A. Khan and F. Algarni, "A healthcare monitoring system for the diagnosis of heart disease in the IoMT cloud environment using MSSO-ANFIS," IEEE Access, vol. 8, pp. 122259-122269, 2020.

[14] R. Ferdousi, M. A. Hossain, and A. E. Saddik, "Early-stage risk prediction of non-communicable disease using machine learning in health CPS," IEEE Access, vol. 9, pp. 96823-96837, 2021.

[15] S. Palaniappan and R. Awang, "Intelligent heart disease prediction system using data mining techniques, in 2008," IEEE/ACS International Conference on Computer Systems and Applications, vol. 8, no. 8, pp. 108-115, 2008.

[16] P. K. Anooj, "Clinical decision support system: risk level prediction of heart disease using weighted fuzzy rules," King Saud Univ.-Comput. Inf. Sci.vol. 24, no. 1, pp. 27-40, 2011.

[17] A. Taneja, "Heart disease prediction system using data mining techniques," Oriental Journal of Computer Science and Technology, vol. 6, no. 1, pp. 457-466, 2013.

[18] J. Sujata and M. K. Nair, "Prediction of heart disease using classification based data mining techniques, in," Smart Innovation, Systems and Technologies, vol. 32, pp. 503-511, 2015.

[19] K. S. Purushottam, K. Saxena, and R. Sharma, "Efficient heart disease prediction system," Procedia Computer Science, vol. 85, pp. 962-969, 2016.

[20] J. K. Kim and S. Kang, "Neural network-based coronary heart disease risk prediction using feature correlation analysis," J. Healthc. Eng.vol. 13, 2017.

[21] A. U. Haq, J. P. Li, M. H. Memon, S. Nazir, R. Sun, and I. Garcia-Magarino, "A hybrid intelligent system framework for the prediction of heart disease using machine learning algorithms," Mobile Information Systems, vol. 21, 2018. 
[22] S. M. S. Shah, F. A. Shah, S. A. Hussain, and S. Batool, "Support vector machines-based heart disease diagnosis using feature subset, wrapping selection and extraction methods," Computers \& Electrical Engineering, vol. 84, p. 18, 2020.

[23] K. Budholiya, S. K. Shrivastava, and V. Sharma, "An optimized XGBoost based diagnostic system for effective prediction of heart disease," J. King Saud Univ. - Comput. Inf. Sci.vol. xxxx, 2020.

[24] B. Martins, D. Ferreira, C. Neto, A. Abelha, and J. Machado, "Data mining for cardiovascular disease prediction," Journal of Medical Systems, vol. 45, no. 1, pp. 1-8, 2021.

[25] S. Barik, S. Mohanty, D. Rout, S. Mohanty, A. K. Patra, and A. K. Mishra, "Heart disease prediction using machine learning techniques," Advances in Electrical Control and Signal Systems, vol. 665, pp. 879-888, 2020.

[26] R. Spencer, F. Thabtah, N. Abdelhamid, and M. Thompson, "Exploring feature selection and classification methods for predicting heart disease," Digital health, vol. 6, pp. 2055207620914777-10, 2020

[27] Y. Khourdifi, M. Bahaj, and M. Bahaj, "Heart disease prediction and classification using machine learning algorithms optimized by particle swarm optimization and ant colony optimization," International Journal of Intelligent Engineering and Systems, vol. 12, no. 1, pp. 242-252, 2019.

[28] P. M. Kumar and U. Devi Gandhi, "A novel three-tier Internet of Things architecture with machine learning algorithm for early detection of heart diseases," Computers \& Electrical Engineering, vol. 65, pp. 222-235, 2018.

[29] S. S. Sarmah, "An efficient IoT-based patient monitoring and heart disease prediction system using deep learning modified neural network," IEEE Access, vol. 8, pp. 135784-135797, 2020.

[30] S. Radhimeenakshi, Classification and Prediction of Heart Disease Risk Using Data Mining Techniques of Support Vector Machine and Artificial Neural Network," in Proceedings of the International Conference on Computing for Sustainable Global Development, INDIACom, 2016.

[31] M. A. Khan, "An IoT framework for heart disease prediction based on MDCNN classifier," IEEE Access, vol. 8, pp. 34717-34727, 2020.

[32] Y. Pan, M. Fu, B. Cheng, X. Tao, and J. Guo, "Enhanced deep learning assisted convolutional neural network for heart disease prediction on the internet of medical things platform," IEEE Access, vol. 8, pp. 189503-189512, 2020.

[33] H. Benhar, A. Idri, and J. L. Fernández-Alemán, "Data preprocessing for heart disease classification: a systematic literature review," Computer Methods and Programs in Biomedicine, vol. 195, p. 105635, 2020. 